\title{
From Euclidean Geometry to knots and nets ${ }^{1}$
}

\begin{abstract}
: this paper assumes the success of arguments against the view that informal mathematical proofs secure rational conviction in virtue of their relations with corresponding formal derivations. This assumption entails a need for an alternative account of the logic of informal mathematical proofs. Following examination of case studies by Manders, De Toffoli \& Giardino, Leitgeb, Feferman and others, this paper proposes a framework for analysing those informal proofs that appeal to the perception or modification of diagrams or to the inspection or imaginative manipulation of mental models of mathematical phenomena. Proofs relying on diagrams can be rigorous if a) it is easy to draw a diagram that shares or otherwise indicates the structure of the mathematical object b) the information thus displayed is not metrical and c) it is possible to put the inferences into systematic mathematical relation with other mathematical inferential practices. Proofs that appeal to mental models can be rigorous if the mental models can be externalised as diagrammatic practice that satisfies these three conditions.
\end{abstract}

At the end of his (2012), Solomon Feferman commits himself to a formalisability thesis about mathematical proofs that he characterises as follows:

...the Formalizability Thesis should be given a very strict reading, namely that (i) every good proof has an underlying logical structure, (ii) that structure is completely analyzed in the derivation that formalizes the proof, and, finally (iii) that derivation assures the correctness of the theorem proved on the basis of the background assumptions expressed by the axioms and rules of the system in which the proof is formalized.

\footnotetext{
${ }^{1}$ I am grateful to conference audiences in Oxford, Paris, Nancy and Brussels for their patience and penetrating questions. Much of the work on this paper took place during my year as a visiting professor at the Vrije Universiteit Brussel, and I am grateful to the directors and participants of the Strategic Research Project on Logic and Philosophy of Mathematical Practices at VUB for their material and intellectual support. Valeria Giordino, Silvia de Toffoli, Irina Starikova and three anonymous referees read drafts and made helpful comments.
} 
There is an argument against ii) that is already well rehearsed in mathematicians' memoires ${ }^{2}$ and the philosophy of mathematical practice literature. ${ }^{3}$ Briefly, the case is that for many, perhaps most good proofs, there is no such thing as the "derivation that formalizes the proof" (because several quite different formalisations may be possible, or there may not even be $a$ "derivation that formalizes the proof" because the proof resists formalisation for reasons described in the detail of the literature (see notes 2 and 3). This paper will assume the success of those arguments and therefore take Feferman's ii) to be false. If ii) is false then so is iii). But what about i)? We might be tempted to abandon it, too, perhaps under the influence of a view such as this:

...there is, strictly, no such thing as mathematical proof; ...we can, in the last analysis, do nothing but point; ...proofs are what Littlewood and I call gas, rhetorical flourishes designed to affect psychology, pictures on the board in the lecture, devices to stimulate the imagination of pupils. ${ }^{4}$

Adopting such a view would solve many problems at a stroke, at the cost of introducing the new challenge of explaining the error of all those philosophers and mathematicians who think that proofs are more than gas, and that proofs have something to do with the reliability of mathematical knowledge. This paper will not take that route.

Rather, we will keep a version of Feferman's i) while denying his ii) and iii). We then have to say something about the underlying structures of proofs. Evidently (remembering the arguments against Feferman's ii)), this view of such underlying structures must allow for all sorts of inferences, not only those that can be rendered in a formal system without distortion. In this paper, we will assume a version of Feferman's i) in which 'logical structure' is understood broadly to include mathematical inferences of any sort, including those that cannot be modelled in formal logic without serious distortion. ${ }^{5}$

2 See for example Manin (1981); Van Bendegem (1988, 1990), who uses this thought as a motivation for philosophy of mathematical practice; Hersh (1997); Rav (1999) on the mathematical interest of topicspecific proof ideas and (2007); Robinson (2000) to make room for the explanatory function of some proofs; Thurston (2006: 48); Auslander (2008); Pelc (2009) to argue the irrelevance of derivations to mathematicians' confidence in theorems; Hales (2012: $\mathrm{x}$ ).

3 Lakatos (1976) predates the formation of the philosophy of mathematical practice as a field. Azzouni (2004, 2009, 2013), Detlefsen (2008), Buldt, Löwe \& Müller (2008), Goethe \& Friend (2010), Larvor (2012a, 2012b, 2016), Leitgeb (2009) seeking to rehabilitate Gödel's allusions to mathematical perception, Macbeth (2015) and Tanswell (2015) all problematise the logical relation between derivations and proofs. For Avigad (2008:306), the difference between derivations and real proofs is the background for reflection on the growing use of computers in mathematics.

4 Hardy (1929) p. 18.

5 This broadening of the conception of logic has some affinity with the heterogeneous logic project of Barwise and Etchemendy, as Shin explained it, "... the heterogeneous logic project aims to expand the territory of logic... Logic is not inherently tied up with sentential representation only." (Shin, S.-J. (2004: 93). 
We therefore require an account of the rational grounds for mathematicians' acceptance of proofs that does not appeal to parts (ii) and (iii) of Feferman's formalizability thesis. However, for the most part, the discussions in the literature do little to suggest how such an account might go. Some of the writers canvassed here refer to mathematicians' understanding ${ }^{6}$ - the idea is that by working through the proof, the mathematician comes to understand that the conclusion is true (even if the proof is not explanatory). This points to an important feature of mathematical proofsthat in working through a proof, the reader engages in the same sort of activity as the writer (namely, mathematics). ${ }^{7}$ This may mark an important difference between mathematics and other kinds of scientific research and scholarship, in which a publication is often a report of activity carried out by the writer but not reproduced or re-enacted by the reader (a physical experiment or an archaeological dig, for example). However, this thought alone cannot answer the need, because all sorts of mathematical activities induce insight aside from proofs. For example, calculating some notable cases of a general hypothesis might induce an aha!-moment, but such calculations do not constitute proof. In any case, a mere feeling of insight has no logical standing in even the broadest conception of mathematical proof. For a proof rationally to convince a mathematician, the mathematician certainly must understand the proof. But what is understood ? ${ }^{8}$ How is this understanding different from the understanding that experts in other disciplines have of the arguments they read? An historian may read a research paper in history with understanding and thereby gain a feeling of insight, but the conclusion of the paper will not have the epistemic security of a mathematical theorem (even if the historian finds it utterly convincing). Similar remarks apply to (nevertheless interesting and relevant) discussions of mathematical skill, such as Löwe and Müller (2010). What is it about the activity in which the mathematician is skilful, that secures mathematical standards of rational conviction?

\footnotetext{
${ }^{6}$ Feferman, in the (2012) article already quoted, argues that diagrams persist in mathematics because they are essential to human understanding of proofs. Suppose this is true; this suggests the possibility of giving an account of the argument-structure of the picture-proofs that he considers without analysing away the inferential role of the pictures. Such an account would contradict Feferman's position, as quoted here.

7 This remains true in the case computer proofs, so long as by 'writer' we mean the programmer rather than the machine. Note, the reader and author of a proof need not engage in precisely the same activities because they may take different routes from one stage to another. One might have to work out in detail some sub-result that the other can see immediately as a special case of some more powerful lemma already known, for example. (I came to appreciate this point after hearing Yacin Hamami make it on the occasion of his doctoral defence.)

${ }^{8}$ As Pollard put it in his review of Hersh (2014), "The real question is how the arguments [in mathematical proofs] manage to present the experts with a mathematical grade of evidence." (2014:273)
} 
To answer this question, we need a model (or more likely, a family of models ${ }^{9}$ ) of mathematical proof to account for the epistemic power of mathematical argument without appealing to derivations in formal systems, and without simply saying that mathematicians can prove theorems because mathematicians have inferential powers.

\section{The Euclidean Diagram}

Fortunately, we do not have to build such a model from nothing. We already have an account of one kind of mathematical proof that explains how such proofs guide and constrain the mathematical activity of the reader so as to secure rational grounds for their theorems. This account appeals only to elements present on the page and to operations on those elements licensed by the mathematical practice in question. There is no reference to a strictly formal deduction that might in principle be given but never is. This account is Kenneth Manders' analysis of Euclidean plane geometry (2008b). Our question, then, is whether the model in Manders' paper, 'The Euclidean Diagram', offers any help with understanding how present-day proofs do their logical work.

'The Euclidean Diagram' is solely concerned with ancient plane geometry, and one might wonder whether it is both too ancient and too planar-geometrical to be of interest, since proof-practices have changed since Euclid's day and diagrams now rarely occur in published proofs, except as illustrations or for heuristic purposes. ${ }^{10}$ Manders himself insists on the value of, "focus on one definite, cogent reasoning practice" (2008a p. 75). Nevertheless, he also says that,

Euclidean diagram use forces us to confront mathematical demonstrative practice, in a much richer form than is implicit in the notions of mathematical theory and formal proof on which so much recent work in the philosophy of mathematics is based; and to confront rigorous demonstrative use of nonpropositional representation. The philosophical opportunities are extraordinary. ${ }^{11}$

So it seems that Manders sees in this case-study the potential to challenge the terms of 'much' recent work in philosophy of mathematics, and his phrase 'rigorous demonstrative use of non-propositional representation' is sufficiently broad to include inferential practices other than the use of Euclidean diagrams. This is the possibility that the present paper sets out to explore. Ideally, we would like to find cases in research mathematics from the $20^{\text {th }}$ century, after the emergence of mathematical logic and set theory. Otherwise, our speculative attempt to extend Manders' approach would be vulnerable to the objection that the mathematicians in our case-studies employed "non-

\footnotetext{
9 Recall Wittgenstein's remark that "mathematics is a MOTLEY [BUNTES Gemisch] of techniques of proof". (RFM III 46:176). Given this variety, we should not insist on seeking a single model to account for all mathematical inferences.

${ }^{10}$ See Carter 2010

${ }^{11}$ (2008a) p. 69. 'Recent' here means 'recent in the mid-1990s', the time of writing.
} 
propositional representation" in their "mathematical demonstrative practice" just because "the notions of mathematical theory and formal proof on which so much recent work in the philosophy of mathematics is based" were not then available.

In the companion piece to 'The Euclidean Diagram' in Paolo Mancosu's volume (2008a), Manders gestures at some 'Tasks for the future' (pp. 75-8). These are mostly confined to ancient geometry. However, he mentions ( $p .76$ ) his work on Descartes' analytic geometry, which takes us into a wider and more recent mathematical world that includes symbolic-algebraic reasoning as well as reasoning with diagrams. Manders' work on Descartes is set out in a paper that he wrote in the 1990s, 'Euclid or Descartes? Representation and Responsiveness', that remains as yet unpublished but which has circulated informally. In this paper, Manders analyses the way Descartes took problems in traditional plane geometry, converted them into his newly invented symbolic algebra, solved the relevant equations and then returned to the traditional practice in order to determine which of the algebraic solutions answers the original question. In the body of this unpublished paper, Manders develops an analytical apparatus that he then applies to the case of Descartes' treatment of ancient geometrical problems, but which is sufficiently general to be applied elsewhere. In the preamble to this analysis, Manders writes,

Philosophers are now faced with the conceptual agility of 20th-century mathematics: hopping a functor to another category any time superfluous details are sensed (homology groups in topology); bringing in details seemingly extraneous to one's question by a representing functor (group representations in the theory of abstract groups); explaining families of simple number-theoretic facts anyone can see one-by-one, by some hard to master "underlying" abstract structure (Fermat's problem). ${ }^{12}$

This remark, together with the general articulation of the analytical apparatus, suggests that the possibility of extending his analysis to present-day mathematics has occurred to Manders, even though this ambition is nowhere explicitly stated. Much of the work of this unpublished paper is to explain in one, historically early case, how it is possible to move between quite different proof-practices without violating the specific rigour of either-in other words, to understand in a relatively simple case the conceptual agility just described. This 'conceptual agility' will be important for what follows.

The plan of this paper is first to examine 'The Euclidean Diagram' with a view to extracting ideas that might be brought to bear on contemporary mathematics - this supplies five questions to take forward to further cases; second, to consider existing case-studies that might prima facie be thought to analyse modern mathematics in something like the spirit of 'The Euclidean Diagram', and ask the five questions about

\footnotetext{
12 Though this paper is unpublished, it is a developed piece of work and benefits from two decades of refinement. We may therefore treat it as a reasonably reliable expression of Manders' view.
} 
them; and third, to argue that appeals to diagrams (and by extension, mental models) in proofs may be rigorous, provided that:

a) it is easy to draw a diagram that shares or otherwise indicates the structure of the mathematical object;

b) the information thus displayed is not metrical; and

c) it is possible to put the inferences into systematic mathematical relation with other mathematical inferential practices.

Obviously, these three general conditions do not guarantee the validity of proofs. Our hypothesis is that these are the principal conditions that the relevant diagrammatic proof-practice must satisfy for the question of the validity of a diagrammatic proof to arise at all. We do not claim that these three conditions are jointly sufficient for a diagrammatic proof-practice to be in good logical order-there may be others as yet unidentified. Nevertheless these three are a significant step in the direction of a sufficient conjunction of conditions. As we shall see, Euclid I-IV satisfies these three conditions a-c.

\section{Logical features of the Euclidean Diagram}

The plane geometry of the first four books of Euclid is the usual first port of call in discussions of proof-practices that are not fully analysable into logical inferences coded in formal logic. This is for the good reason that here we find a body of mathematics organised logically, in the sense that it starts with definitions and postulates and is built up by proofs, but its proofs cannot be fully analysed without distortion using a formal logic of propositions because operations on diagrams play an essential role in the proofs. The core of Manders' (2008b) analysis is his distinction between exact and coexact information found in diagrams. Exact information is such matters as equality of lengths and angles, proportions, parallelism and curvature. Co-exact information is things like the inclusion of one area inside another or the existence of an intersection of lines. Exact information is vulnerable to deformations of the diagram resulting from careless drawing (the tomato-shaped circle or the triangle with curved sides), or accidental features such as a general triangle being drawn to look isosceles. Co-exact information is not so vulnerable, and the vital point is that when Euclid's proofs take information from a diagram, the information they take is always co-exact. That is how it is possible to make good arguments on bad drawings, and how it is possible to draw general conclusions from particular diagrams.

Manders' work on Euclid is well known, so I shall not elaborate it further but will make five unoriginal observations that have a bearing on the argument of the rest of the paper.

First, these proofs consist of diagrams with text and they rely on inferences in the text as well as co-exact information taken from the diagram. These are not proofs without words; nor are they discursive proofs with illustrations. Rather, the diagram and the 
text work together. There is typically a constructive phase, when the diagram is built up using moves that are either specified in the postulates or have been shown to be valid in earlier proofs. Then, co-exact information is read from it and fed into a textual argument. With an eye on case-studies later in this paper drawn from knot theory and low dimensional topology, it's worth noting that these changes to the Euclidean diagram are constructions, not manipulations.

Second, these constructive moves (adding lines or circles to the diagram) are inferential actions. ${ }^{13}$ They are strictly controlled by the first three postulates-nothing else is permitted. ${ }^{14}$ We start with some conditions, which we realise in a diagram. We then do something (carry out permitted constructions of lines and/or circles), at the end of which we have some more information, and the information we gain is reliable because the activity was governed by rules that ensure that it will not lead us astray (these rules are the postulates plus the restriction to reading off co-exact information). This rigour is why ancient geometry is reliable (Manders puts it more trenchantly, "Euclid... Apollonius and Archimedes are virtually without error" 2008a p. 67). This yields a criterion for evaluating other candidate-cases of non-propositional inferential actions. Namely, it should be possible to do for such a candidate what Manders did for the plane geometry in Euclid, that is, specify the implicit rules that render the practice rigorous as mathematical proof. The claim of the present paper (that such practices should be public, non-metric and systematically relatable to other mathematics) is a step in this direction. To quote Manders again, "At its most basic, a mathematical practice is a structure for cooperative effort in control of self and life." (2008b p. 82, emphasis in original). In looking for more of the same, we should look for shared proof-practices governed by rules that ensure reliable results - not every napkin-diagram or thoughtexperiment will qualify. This is a (defeasible) reason for considering published proofs rather than spontaneous mathematical expressions. Moreover, the language in Euclid is

\footnotetext{
13 I have elaborated and defended the notion of inferential actions in Larvor (2012a). An inferential action is anything one might do that contributes to the logic of an argument. Such actions include speech-acts, manipulations of matter (such as physical experiments), taking of measurements, manipulation of physical or digital models, calculations, operations on propositions (such as applications of logical rules like modus ponens), bodily performances and in mathematics, a host of topic-specific moves in the sense of Rav (1999). Proofs are full of imperatives that instruct the reader to build, change, move, identify or otherise re-order all sorts of objects, including but not only, propositions. What matters is that such actions be governed by (explicit or implicit) norms. If the aim of logic is to understand the difference between valid and invalid inferences, then these actions fall within its scope, even though we then lose any prospect of a single unifying theory of logic. Since formal mathematical logic already includes a multitude of systems with varying symbolisms and logical strengths, with extensions designed for the analysis of modal, temporal and deontic arguments, this is no great loss.

${ }^{14}$ With the exception of Elements I.4, where a triangle is rigidly displaced. This is well known to commentators (see, e.g. Ferreirós 2016 p. 130n20, or Russell 1902, who calls superposition "a tissue of nonsense"). Euclid seems to have wished to avoid superposition wherever possible. In any case, superposition is still a highly disciplined move, even if it does not fit the constraints of Euclid's overall practice.
} 
very restricted and formulaic, because the permissible actions have been reduced to a small and standard list.

Third, traduttore traditore! 15 Goethe is said to have observed that, "mathematicians are like Frenchmen; if one speaks to them they translate it into their own language, and then it will be very soon something entirely different." Discounting for xenophobia and hyperbole, Goethe was right. Mathematical analysis of some natural or social phenomenon requires abstraction, the discarding of myriad details and it (usually) also requires a systematic notation or representation that facilitates the analysis together with conventions to make the notation work. The point for the present purpose is that this is also true within mathematics. When a problem or a question is translated from one mathematical idiom to another (or-what can come to the same thing-shifted into a new domain), it becomes something different while (hopefully) retaining whatever structure it is that we hope to understand. To continue the quotation from Manders, speaking of the giants of ancient Greek mathematics, “...their every result has a counterpart in modern mathematics, even if subsumed in patterns of claims and proofs recognized much later... The Pythagorean theorem lives on in Euclidean and even Riemannian metric spaces." When we consider the theorems, it may be tempting to think that there is little difference between ancient and modern geometry-rather, the differences become vivid when we consider the nature of the inferences. A logical reconstruction in the style of Hilbert cannot account for the successes of ancient Greek mathematics, because the modern version deploys inferential machinery that was not then available, and strips out the inferential practices that constitute proof in that context. Worse, it finds fault where there is none. For example, there is no need to prove that there is a point of intersection where two lines cross, because crossing Euclidean lines cannot fail to have a point of intersection. There is no possibility of a line on a Euclidean diagram representing a dense-but-not-continuous set because there are no such things in this mathematical practice. ${ }^{16}$ Rather, lines and their intersections are the fundamental Euclidean elements. They do not represent some other, more finegrained level of mathematical reality such as the real numbers as we now conceive of them, or any subset thereof. ${ }^{17}$

For studies of other, more recent proving practices, this point (traduttore traditore) has two consequences. One is that it explains why mathematicians move problems from one domain to another. A well-chosen translation can make more information available, or it might dispose of superfluous information. It may also change the range

15 Literally, 'translator, traitor', meaning that all translation traduces (except that the Italian original has a play on words that cannot be reproduced in English).

16 More precisely, to take the case of I.1, that the circles intersect is co-exact information because it is unaffected by bad drawing - however ovoid or tomato-shaped your circles are, in drawing the second one, you have to start inside the first, go outside the first in order to pass the other side of the centre of the second circle, and then re-enter the first circle again. This robustness is what rigour means in this context. (Manders 2008a p. 69)

${ }^{17}$ For the historical context of this point, see Ferreirós (2016) chapter eight, and most especially p. 213. 
of actions available to the mathematician. ${ }^{18}$ The second consequence is that in examining the logic of a proof, we should look at the proof in the form and the idiom in which it is used, rather than first translating it into some other idiom. It may be that the proof employs inferential moves that are possible only using precisely that kind of representation, governed by precisely those rules. The Euclidean inferences that Manders analyses are possible only by using Euclidean diagrams and following Euclidean rules. Other kinds of inscription governed by other rules support different inferences, even if the theorems look similar.

Fourth, there is in Manders' analysis no appeal to ineliminable intuition. We take the co-exact information directly from the diagram, by looking at it with selective attention, but there is nothing mysterious about this and no reason in principle why it might not be automated. Indeed, Avigad, Dean, and Mumma (2009) have developed a formal system based on Manders' reading of the Elements, though, as the authors note, it does not emulate the spatial reasoning in ancient geometry, so the inferences in their system are not the inferences in Euclid, even if the results correlate. Of course, any automation of a human proof-practice runs into our third point (traduttore traditore!). It may be that preparing some given proof practice for automation changes it so that the inferential actions are no longer recognisable from the original, but this is a matter of the detail of the system used and the available technology. In their paper, Avigad, Dean, and Mumma conclude by noting some differences between the Elements and their system, and reflecting on what automation would involve in this case. The general point is this: one might think that the rising successes of automated proof finders and checkers would present a problem for an approach to proofs rooted in the philosophy of (human) mathematical practices. This is not so. Manders claims that some proofs involve actions on objects other than propositions - there is no reason in principle why these actions should not be carried out by machines. ${ }^{19}$ The diagrams could be drawn in dust, or on a computer screen, or stored virtually, and the co-exact information could be extracted in any number of ways other than by human sight. This is why Manders need not draw on cognitive science or the psychology of perception in his logical analysis of Euclidean inference-making. The inferential actions in Euclidean diagram-use have to be possible for humans, but they need not be performed by humans and their logic does not depend on the specifics of human cognition.

\footnotetext{
18 See Starikova (2010) for a case study on this point.

19 Interestingly, Alan Weir, developing a defence of formalism against criticism based on the difference between real proofs and proof-theoretic derivations, makes a similar point, "I did not require that the formal rules of a derivation be what we would recognise as logical rules." (2016:30). He then lists all manner of rules for actions that we might count as inferential: "basic rules for differentiation, power rules, product rules, quotient rules, the chain rule and so on." Both he and we note that automation of proofs need not be, and probably cannot be, the encoding of proofs in some version of formal logic. Rather, what is automated is a much wider class of inferential actions, including topicspecific actions on objects other than propositions (or sentences).
} 
Finally (fifth), note that Manders' argument that proofs in Euclidean geometry make essential use of non-textual inferential actions depends on the role in the practice of diagrams. Many of the most plausible candidates for Manders-style case-studies of contemporary mathematics appeal to uses of diagrams or other non-textual representational means (Cayley diagrams, commutative diagrams, knot diagrams, and so on). This is because the distortion involved in translating a proof into a derivation in formal logic is especially clear and salient when the proof includes visual reasoning. For example, Tanswell (2015) chooses the mutilated chessboard problem precisely because the visual reasoning is quick and clear, and the corresponding formal proofs (there are several quite different versions, so we cannot speak of 'the' formalisation of this visual argument) are significantly longer and more complicated. In such cases, the claim that the translation is not routine, and that the inferences in the formal derivation really are not tidied up versions of the inferences in the visual argument is especially compelling.

However, as we shall shortly see, not every putative case of non-propositional inferential action depends on the use of diagrams. In some proofs, we seem to work directly on the mathematical objects themselves. Think, for a simple example, of the standard proof of the infinity of primes. Here, the objects of the imperative verbs are not diagrams or symbols but rather the numbers themselves (take the members of the assumed finite set of primes, form the product, add one, etc.). In that case, it is easy to write the proof down because we have systematic notations for arithmetic-even in natural language. More generally, the vital feature in these cases is not that they are visual, or non-propositional, or iconic. Rather, the feature that prevents non-distorting translation into a general logic is the topic-specificity of the inferential moves. Euclidean inferences are possible only within something like Euclidean practice. Considerations of divisibility only arise in domains that share enough structure with the natural numbers for the question of whether one element divides another to be meaningful and informative (so in a field, for example, it's meaningful to ask whether one element divides another but uninformative because the answer is always 'yes'). Mathematical induction is available in some domains but not others. And so on. Sometimes, these topic-specific (or better, non-universal) inferential moves are coded into representational systems. Ordinary high-school algebraic notation, for example, has the non-universal move of multiplying out brackets coded in. The non-universality of the moves available in some notational or diagrammatic practice is what matters for this argument. This is what Euclidean diagram-use has in common with other inferential practices that resist translation into formal logic, whether they employ diagrams or not. $^{20}$

In terms of Feferman's formalizability thesis, we can read Manders' work as unearthing the 'underlying structure' of Euclidean plane proofs. His analysis shows that it is possible in at least one case to affirm our version of part i) of Feferman's formalizability

20 I make this argument in greater detail in Larvor (2012a). 
thesis while denying parts ii) and iii). What we take from Manders for application elsewhere are our three criteria.

The first, (a) is that mental models must be externalisable as diagrams (or in some other publicly sharable way such as physical or computer models). At its core, this is just the requirement that proofs should be available for public scrutiny. We focus here on twodimentionsal diagrams because they have been central to mathematical practice for most of the history of mathematics, but there is nothing essential about this. Some early modern editions of Euclid were pop-up books with fold-out models of polyhedra, and digital technologies now offer all sorts of new possibilities in this respect. All that matters is that the representations should be available for public examination. Moreover, it is advantageous if they are open to easy manipulation and (re)construction. This explains the continuing popularity of chalk and white boards among mathematicians. By 'easy' here, we mean only that there should be no requirement that mathematicians should be especially good at drawing or making paper models. What may not always be easy is working out what to draw in order to represent the mathematics effectively. ${ }^{21}$

The second criterion (b) is that the information gained from such diagrams should not be metric (so that the inferences are not vulnerable to local deformations ${ }^{22}$ ). This is a wider notion than Manders' co-exactness, which he devised for the specific case of Euclid. For example, the fact that a minimal tree of a planar graph has one more vertex than it has edges is a non-metric feature that one might see from a diagram, but it falls outside Manders' notion of co-exactness. Similarly, subitising small numerosities and spotting patterns in dots are not vulnerable to local deformation, but are not co-exact in Manders' sense. ${ }^{23}$

Finally, (c) it should be possible to relate the diagrammatic inferences in some systematic way to other mathematical proof-practices. ${ }^{24}$ This condition is Manders' observation about conceptual agility, put to a new purpose. He presents it as a challenge for philosophers; here we offer it as a criterion of good order for mathematical practices. What Manders shows in his unpublished paper is how Descartes was able to relate Euclidean diagrammatic practice systematically to symbolic algebra. Such connections are only possible with stable, orderly, rule-governed representational practices. Contemporary mathematics is rich in such connections, so much so that a single proof might contain many different inferential practices expressed

\footnotetext{
21 I am grateful to Robert Thomas for this distinction, and for pointing out my arbitrary restriction to twodimensional representations.

22 I am grateful to an anonymous referee for this way of putting the point.

${ }^{23}$ Silvia De Toffoli, in an unpublished presentation, has taken some steps towards classifying diagrams that can support inferences. Her principal criterion is the Jordan curve theorem. On the significance of the Jordan Curve theorem for reasoning with pictures, see also Corfield 2003 p. 257.

24 This criterion was originally inspired by some remarks in De Toffoli and Giardino (2014) about the connections between knot diagrams and other notations.
} 
in different mathematical idioms. Moving between such diverse practices is the 'conceptual agility' that Manders' mentions in his unpublished paper. He is not the only philosopher to remark on the importance of moving between mathematical practices; José Ferreirós makes this the theme and title of his recent book (2016). For Ferreirós, it is the interplay between mathematical practices that gives mathematics its stability, order and meaning. It is only by considering this interplay that the 'naturalness' of structures like the natural numbers can be undersood. Similarly, Emily Grosholz argues that mathematicians typically require a 'consortium of modes of representation' (2007 p. 235) in order to make mathematical sense. She offers many examples from advanced mathematics in her final chapter, but one of the simpler ones will give the flavour. She cites a textbook on topology and geometry, and notes that the authors illustrate the concept of the Cartesian product of two sets with the Euclidean ${ }^{25}$ plane, which they characterise both as $\mathbb{R} X \mathbb{R}$ and with the aid of a diagram. According to Grosholz, the diagram is there because the Euclidean plane is a 'canonical object' that will appear later in the textbook, and as such it is not merely the product of the set of reals with itself. Rather, it is this product treated as a vector space with a natural inner product that induces a norm, which makes it a metric space and ultimately a topological space. The diagram is there to help make sense of the algebraic and topological definitions that would otherwise be arbitrary and perhaps even unintelligible.

Ferreirós and Grosholz show (in different ways, within different philosophical research programmes) that contemporary mathematics is a ramifying web of interrelated (or in Ferreirós' terms, interplaying) practices and modes of representation. This is characteristic of mathematics - the suggestion here is to make this feature into part of a criterion of mathematical practice in good order. For a trivial example, multiplication with sticks (so-called 'Japanese multiplication') can be shown to work by relating it to other multiplication procedures in other modes of representation (such as long multiplication using Hindu-Arabic numerals). For a historical example of this criterion at work, consider the Argand diagram. Until Argand published his paper, calculating with imaginary numbers was a mathematical practice in dubious standing. The Argand diagram made imaginary numbers respectable by relating the practices involving them to another part of mathematics that was known to be in good order, namely, algebraic geometry. Nowadays, it is more often the other way around: a diagrammatic practice gains respectability by standing in a systematic mathematical relation with nondiagrammatic mathematics. For example, consider Cayley graphs of finitely generated groups. These diagrams might, in some alternative history of mathematics, have been a free-standing bit of mathematical practice. One can imagine a community of mathematicians who thought about groups entirely in terms of and by means of Cayley graphs. However, this would be an impoverished practice with no way of deciding those questions about finitely generated groups that cannot be settled by inspecting and manipulating Cayley graphs. It is precisely because we have notations and inferential

\footnotetext{
25 'Euclidean' in a modern topology textbook does not mean, of couse, what it means when we discuss
} Euclid's Elements. 
practices for algebraic group theory and for graph theory, that Cayley graphs are definitely part of mathematics (otherwise they might have been on the margin of mathematics, like chess-problems) and both useful and interesting. For a trivial example, on a Cayley graph it is very easy to see whether the group is commutative, but communtativity is a question that arises naturally with the use of algebraic notation.

\section{What has already been done? Case studies of recent mathematics}

Having sketched Manders' analysis of Euclidean diagrammatic inferential practice and drawn our three criteria out of it, the next task is to look for existing attempts to extend it to present-day mathematics, or otherwise to look for philosophical analyses of mathematical proof that seem to be in a similar line. Direct citation of Manders' articles is, unsurprisingly, dominated by discussions of ancient Greek geometry. Notably, there are in print no attempts to refute his analysis of the logic of Euclidean plane geometry. The experts seem to be convinced by it, and this is a further reason for choosing it as a model for analysis of recent mathematics. Otherwise, his work is mentioned in scholarship on Kant's account of space, work on visual reasoning in general and discussions of Avigad, Dean, and Mumma (2009). There are not many candidates for the role specified, namely, Manders-style analyses of mathematical proof-practices from this or the previous century. Significant exceptions are De Toffoli \& Giardino (2013, 2014, and 2016) and Leitgeb (2009). ${ }^{26}$ These do not explicitly pick up Manders' framework with the intention of turning it into a programme. However, they do take up similar lines regarding the logic(s) of mathematical proof(s) and they are informed by his work.

\section{De Toffoli \& Giardino}

De Toffoli \& Giardino argue by cases that in knot-theory and low-dimensional topology, we meet examples of inferential practices that ineliminably involve non-propositional representations, that are accepted as proofs by the relevant expert communities and that are rigorous because they impose precise constraints on what moves are permissible. In their most recent paper (2016), they present Alexander's (1923) proof that every knot can be represented (not uniquely) as a closed braid (p. 40). They explain the significance of this by presenting the braid groups and some examples of reasoning using braid diagrams. Since Alexander published his proof in the 1920s, it (narrowly) meets our requirement for cases dating to after the emergence of the contemporary foundational language(s) and logical apparatus(es).

The usual proof of Alexander's theorem relies on our ability to imagine manipulating rope. It invites us to imagine a knot lying spread on a plane surface (though not in a

\footnotetext{
${ }^{26}$ And Starikova (2010). Her work on geometric group theory is in the same line but raises problems that lie beyond the scope of this paper.
} 
plane because there are crossings where the rope passes over itself). ${ }^{27}$ The core of the proof is to show that every knot can be given an axis. An axis is a line perpendicular to the plane of the knot but not intersecting the knot itself, such that an ant walking along the length of the rope-loop would always go round the axis in the same direction, either clockwise or anticlockwise. This is not the case for any arbitrary knot, but it is easy to see that it is always possible to transform an arbitrary knot in such a way that it gets an axis without changing its character as a knot. ${ }^{28}$ This is done by successively moving sections of the knot from one side of the chosen point to the other, either simply by sliding or by flipping. Once we have transformed our knot in such a way that it has an axis, then we can turn it into a braid by cutting along a straight line in the plane starting at the axial point. De Toffoli \& Giardino claim that "the reasoning involved in this specific proof cannot be identified exclusively with propositional reasoning, and even less with formal reasoning." (p. 43). Rather (to impose Manders' language on De Toffoli \& Giardino) it is a "rigorous demonstrative use of non-propositional representation". They report that this is not a mere illustration - this invitation to imagine handling rope is essential to the proof. What is more, they claim that this is not a solitary case; rather proofs of this sort constitute a stable inferential practice with strict rules shared among a community of experts. They emphasise the dynamic character of the proof-it does not invite us to look at a diagram, but rather to imagine shifting and flipping sections of rope. Indeed, they point out that "The original proof contains no pictures." (p. 40). It relies on what they call a "cognitive capacity [of] manipulative imagination" (ibid.). ${ }^{29}$

In the previous section, we identified five features of Euclidean diagram-use as analysed by Manders. What happens if we look for these features in Alexander's proof as presented by De Toffoli \& Giardino? The first three points transfer easily. The proof involves text as well as exercises of manipulative imagination-most notably, it includes imperatives as well as propositions (first point). The imagined manipulations are inferential actions (second point). It is certainly the case that a translation of this proof into a formal idiom would change it out of all recognition - the inferential actions involved in a formal version would be different actions on different objects (third point). The actions possible on strings of symbols are quite different from the actions possible on string.

Our fourth claim about Euclidean diagram-use is that there is in Manders' analysis no appeal to ineliminable intuition. Is this true in the case of Alexander's proof, as written up by De Toffoli \& Giardino? The vital word here is 'ineliminable'. There is no doubt that the proof as presented relies on our spatial intuition. We could try to dispose of the appeal to inner intuition by replacing the mental rope with physical rope. Then, it seems that there is no slide into psychologism and the rules of the practice are easy to

\footnotetext{
${ }^{27} \mathrm{~A}$ 'knot' here is a closed loop, which may be tangled, such as the trefoil knot. None of the useful knots known to sailors and fishermen are 'knots' for this purpose.

28 That is, its ambient isotopy class.

${ }^{29}$ See Giardino (2017) for a further elaboration of this notion.
} 
specify: the permitted actions in rearranging a physical knot to give it an axis are those that do not damage the rope. The difficulty lies in the leap to generality. The claim of this theorem is that any knot can be so rearranged, and we do not have-De Toffoli \& Giardino do not give us-an account of the logical process by which manipulating some ropes, either in thought or in the hand, proves that it is always possible to rearrange any knot around an axis. They simply report that "it is left to our intuition to prove that this transformation [into a knot with an axis] is always possible and is not an infinite process" (p. 44). This is remarkable given that by 1923, it was well understood that spatial intuition is not generally reliable. Yet, Alexander had no qualms about relying on it in this proof. The justification of a general result, remember, was one of the central questions about Euclidean diagrams: how do operations on a specific diagram prove a theorem about all diagrams of this sort? Manders goes to the trouble of distinguishing exact from co-exact information and working through the details of case-distinctions (and more) because it does not suffice to say that the Euclidean geometer sees the truth of the theorem in virtue of his or her spatial intuition. The traditional (that is, midtwentieth century) view of diagrammatic arguments was that they are unreliable as proofs precisely because they depend on spatial intuition (which familiar problem-cases show to be unreliable). Manders seeks to refute the traditional, disparaging view of diagrammatic proof (at least as regards Euclidean plane geometry) precisely by removing the appeal to unanalysed spatial intuition from his logical account of how this activity constitutes mathematical proof.

In this connection, it may matter that rather than speak of shifting and flipping, Alexander gives a rule for dividing the rope up into straight sections and replacing each problematic section with a pair of connected straight edges so that the axis falls within the triangle of these three lines (this move is called an 'elementary isotopy'). These two operations (making triangles and shifting + flipping) are mathematically equivalent, but dealing solely with straight edges constrains the possible moves. Recall our contrast between constructions and manipulations; Alexander's procedure is a construction followed by a deletion, so it is closer to Euclid than is De Toffoli \& Giardino's version of his proof. It is also much easier to externalise as a drawing (though shifting and flipping makes a better video).

The last of our five points about the Euclidean diagram was to ask whether every case of non-propositional inferential action depends on the use of a non-textual representation of mathematical content. In this case, we noted that Alexander's paper did not include a diagram. De Toffoli \& Giardino describe the proof as a manipulation of a 'mental model' (picking up Thurston's phrase, p. 44). To ask what mental models are and how they work would be to wade into the debates in philosophy of mind and cognitive science which are beyond the scope of this paper. Besides, we just argued that the reference to mental models need not indicate a problematic psychologism in this case because the mental model can be replaced by diagrams. This marks an important feature of this area: the 'knots' are closed loops with finitely many tangles, so it is always possible in principle to make a diagram (though not always easy). In other 
words, the mathematician can externalise the mental model as a drawing without changing the proof (because the same actions are now performed on the diagram rather than the mental representation). De Toffoli \& Giardino note that, "The practice of proving that is common in this branch of mathematics is in fact quite distant from that of almost every area because it largely relies on 'seeing' topological objects, which amounts to envisaging transformations on the representations available for them." (p. 45). The finitude of the knots is one of the reasons for that distinctness.

On the other hand, the proofs rely on the fact that human spatial intuition is a good judge of the topology of relatively simple finite structures, and such judgments are found elsewhere in mathematics. It is the topology of the Euclidean diagram that represents the co-exact information. In a rather more recent example, simple topological objects are used for calculations in higher dimensional algebra. ${ }^{30}$ It's notable that spatial intuition is much more important in this paper (2016) than in their (2014), because the earlier paper focussed on the usefulness of knot-diagrams, and how these can be used inferentially using (for example) colourability or by using Wirtinger presentations to associate diagrammatic argument with algebra. ${ }^{31}$

Thinking about the use of diagrams in mathematical inferences leads to the use of imagination, partly because the use of diagrams requires some work of the imagination-one might imagine part of the diagram moving or growing or shrinking, for example. One may have to imagine the diagram drawn more precisely than it is in fact. In any case, mathematicians do not always bother to draw the pictures that they have in mind (as in the case Alexander's proof). Reflection on the contrast between De Toffoli \& Giardino's papers of 2014 and 2016 suggests the following: where mathematicians and others talk of 'mental models', they are really talking about internalised diagrammatic practices (just as reliable mental arithmetic is internalised pencil-and-paper arithmetic). ${ }^{32}$ If that is right, then in order to show that a proof that seems to appeal to a mental model is in good logical order, we have to identify the corresponding diagrammatic practice, show that criteria a-c are true of it and then carry out a Manders-style analysis. To explore this thought, we turn now to a philosopher and mathematician who explicitly defends the use of mental models in proofs.

\footnotetext{
${ }^{30}$ See Corfield (2003) chapter 10.4. I am grateful to an anonymous referee for drawing this to my attention.

31 This point, about the relationship between knot-diagrams and other, more discursive sorts of representation, was the the inspiration for our criterion $\mathrm{c}$ ).

32 As we saw with Euclid, another way of examining the role of intuition in a proof is to try to code it into a computer. There is at present no known way of doing this with Alexander's proof. Movies are another option. Some of the illustrations in De Toffoli \& Giardino (2016) are stills from an animation by Ester Dalvit.
} 


\section{Leitgeb}

In his (2009), Hannes Leitgeb aims to rehabilitate Gödel's claims that (some) mathematical laws, axioms and propositions can be in some sense perceived to be true (p. 275). In preparing the ground for this, he argues for a distinction between formal and informal proofs. He emphasises the arbitrariness of the conventions required to reconstruct a piece of informal mathematics in a formal system. In particular, he suggests that the mathematical content of a proof does not usually fix the order of the logical system (p. 269) required for its reconstruction. ${ }^{33}$ He goes on to make some further points that have become familiar in the literature regarding the differences between formal and informal proofs: mathematicians read mathematical notations and representations as meaningful (rather than syntactically, as symbol-strings in derivations), and they regard theorems as true (rather than 'true' or true-in-X). Insofar as mathematical practice involves axioms, the axioms do not play the roles that they play in logic textbooks. Even regarding the axioms of set theory, Leitgeb says that "...group theorists, topologists, and probability theorists usually do not even know these set-theoretic axioms - the set theory they use is likely a version of naïve set theory-and it is not conceptually essential to their proofs that they rely on an axiomatic system of set theory." ${ }^{\prime 34}$ (p. 271). In short, Leitgeb is on the same page as the mathematicians and philosophers we canvassed at the outset in footnotes 2 and 3 . Note that substantiating the last part of this claim ("it is not conceptually essential to their proofs that they rely on an axiomatic system of set theory") would require a logical-Leitgeb might prefer to say 'conceptual' -analysis of the informal proofs in question, in which axiomatic set theory did not play an essential role. Here again, we meet a view about proofs in mathematical practice waiting for someone to carry out work corresponding to Manders' reconstruction of Euclidean proof-practice.

With these motivating considerations in place, Leitgeb distinguishes (what he calls) the semantic and intuitive components of proofs. By 'semantic', he means the combination and manipulation of meaningful propositions. By 'intuition', he means "manipulating and inspecting the mental constructions that are similar to [the relevant mathematical] structures" (p. 278). He offers two examples of such mental constructions. The first is a mental representation of an unlabelled graph. Rather helpfully for the present argument, Leitgeb claims that we can understand his mental representation by contemplating this picture:

\footnotetext{
33 Tanswell (2015) makes a similar argument, that informal proofs underdetermine the details of their formal reconstructions so radically that the former cannot be meaningfully identified with the latter.

34 (p. 271). Leitgeb offers no empirical grounds for these claims about what mathematicians do or do not know. At present it is normal in the philosophy of mathematical practice to make remarks of this sort on the basis of one's standing as a mathematician or acquaintance with mathematicians. Therefore, this is not a complaint about Leitgeb, but rather a point about the state of the sub-field. Besides, other published versions of this claim (by, for example, Kreisel) have not provoked rebukes or rebuttals from other mathematicians.
} 


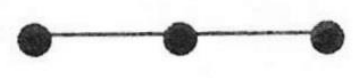

The corresponding mental construction is more faithful than this diagram to the mathematical structure it represents, because the printed version necessarily has a lot of extraneous features (length, thickness and shape of edges, size of nodes, colour, etc.) that the mental construction can do without. His second example is an unlabelled graph with the structure of the natural numbers:

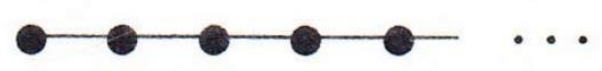

again, we are to think of a mental construction that resembles this diagram but is more faithful to the mathematical object it represents (according to Leitgeb, the mental model is not a copy of this picture before the mind's eye but is rather the fixed point of the operation of chopping the leftmost node off pictures like this). His suggestion, then, is that proving theorems includes inspection and manipulation of such mental constructions. In other words, Leitgeb suggests that proving (sometimes) involves inferential actions (including inspection) on objects other than propositions. Such actions are likely to take place at the points in a proof where a strictly propositional analysis would suggest that there is a logical gap or an implicit appeal to an axiom. What fills the gap or does the work of the axiom, according to Leitgeb, is intuitive (that is, quasi-perceptual) access to a mental construction. This seems very similar to the appeal to mental models that we encountered in De Toffoli \& Giardino, except that they emphasise the dynamics aspect (the 'manipulative imagination') whereas Leitgeb, guided by Gödel's perceptual language, emphasises inspection over manipulation.

It bears repeating that all these writers, Leitgeb, De Toffoli and Giardino (and certainly Gödel) are well aware of the history of logic and mathematics, and of the fall from grace of spatial intuition and diagrammatic reasoning. They know the received view of visual reasoning in mathematics. They also know that logic is distinct from psychology. Nevertheless, attention to practice drives them to claim that mental models have a role in the making and checking of proofs. If they are on to something then it must be 
possible to explain how such proofs are sharable ${ }^{35}$ and rigorous. The ambition of our conditions a-c is to offer a framework for such explanations.

Unfortunately, Leitgeb's discussion does not include any examples of proofs that depend on the sorts of intuitive access to mental constructions that he describes. What his paper omits (perhaps because it originates in a discussion of Gödel) is the thought that the choice of picture depends on what one is trying to prove as well as on the mathematical object that the proof is about. For example, one might think of the natural numbers like this:

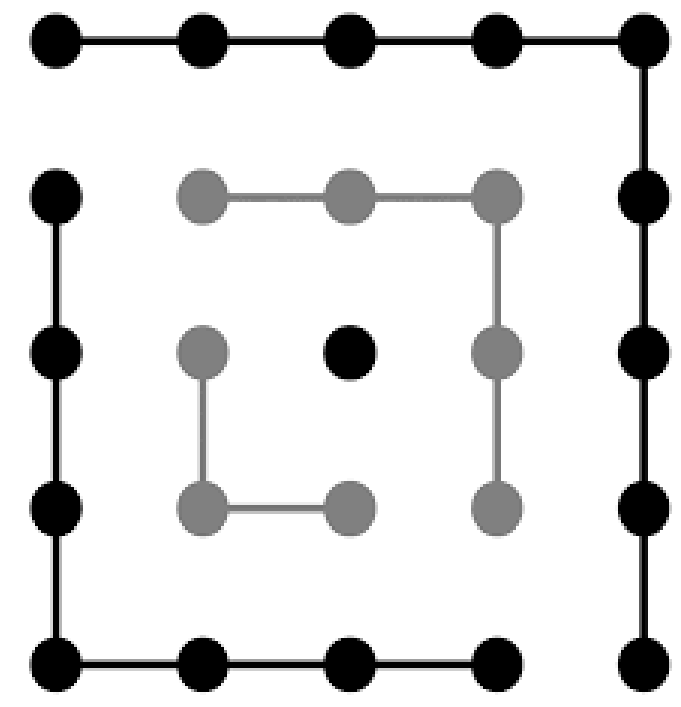

${ }^{35}$ Accounts of meaning that ground the intelligibility of speech and thought in social practice (such as Wittgenstein's anti-private language argument or Vygotsky's social-developmental theory) might suggest stronger versions of point (a). 


\section{Or like this:}

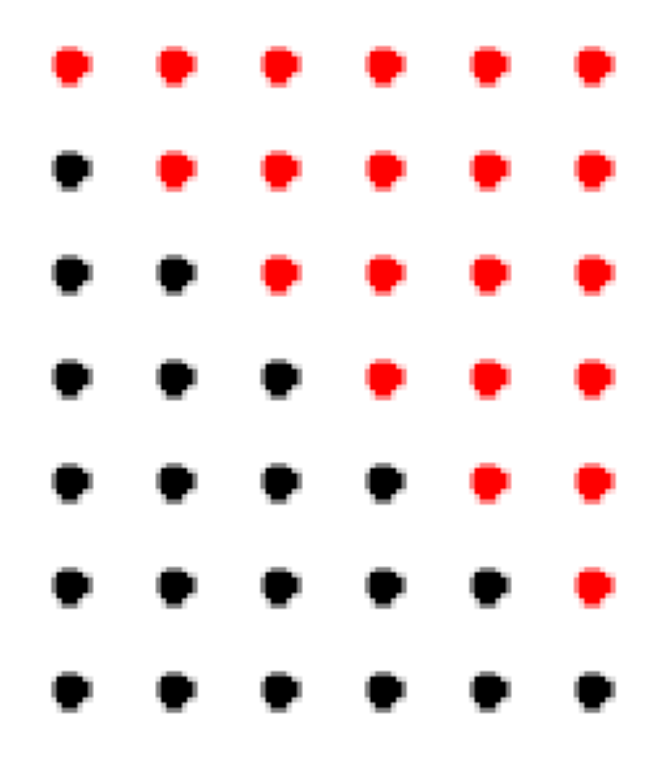

Thinking of the appropriate spatial representation of the relevant mathematical structure is a practical skill that does not appear in Leitgeb's discussion.

\section{Infinite diagrams}

So far, the only proof we have examined in detail is Alexander's, and this is from a field that (as we noted) is concerned with finite knots and braids. Much of mathematics concerns infinite structures, so to be philosophically interesting, our approach has to explain how access to a mental construction (which, being in a human mind, is presumably finite) allows us to prove theorems about infinite structures. Leitgeb takes a step in this direction, in that his second figure and the instructions on how to read it are supposed to make plausible his claim that we can inwardly intuit truths about the natural numbers. However, his paper does not include any sample proofs. This matters, because as noted, the choice of representation is likely to depend on what one is trying to prove. The arrangement of dots at the end of the last section gives the formula for the sum of natural numbers up to some $n$ more or less at a glance, for someone who has been inducted into the relevant mathematical practices. On the other hand, one might entertain something like Leitgeb's image while thinking about the Peano axioms. The dot-proof of the sum of whole numbers formula satisfies our conditions a-c: it is very easy to draw, it does not exhibit metrical information (it is not sensitive to local deformation), and it can be easily related to general arithmetic. We can easily move between dot diagrams and algebraic notation. It is an example of why we had to expand Manders' 'co-exact' into 'non-metrical' in formulating b). It also illustrates one 
way that a finite diagram can prove a result about an infinite structure. The dotdiagram in this case is a rectangle $n$ by $n+1$ with some device to divide it into two 'staircases' from 1 to $n$. Anyone who understands it can see that it does not depend on the choice of $n$.

This is a rudimentary example from ancient mathematics. In the paper already cited, Feferman (2012) offers three cases from modern mathematics: the Cantor-Bernstein theorem, the strong amalgamation theorem and the exact sequence of homology theorem. Feferman's claim with respect to these cases is psychological rather than logical, namely, that it is humanly impossible to understand what these proofs are about without the help of their respective diagrams. This is altogether plausible, but it concerns the understanding of the mathematician rather than the logic of the proof. $\mathrm{He}$ does not provide the logical analysis ${ }^{36}$ of the diagram-use that we need-but it is noteworthy that in his three cases, points a-c hold. What is, in Feferman, an account of how diagrams drawn on finite materials can permit understanding of theorems about infinite structures, can convert into an account of how such diagrams figure in the logic(s) of the proofs. In all of Feferman's cases, the iterative nature of the generative processes mean that it suffices to contemplate a finite section of the infinite structure in order to see what happens to the whole.

\section{Conclusion}

We began by considering Feferman's three-part formalisability thesis. Much literature in the philosophy of mathematical practice argues, effectively, for keeping a version of Feferman's i) with a suitably modified reading of 'logical' while denying his ii) and iii). This requires some alternative to the derivation-based analysis of the arguments in proofs. Ken Manders 'The Euclidean Diagram' offers a case in that fulfils this requirement. In this case,

a) the diagrams are easy to draw and work on (they require no special skill in draughtsmanship);

b) the information thus displayed is not metrical; and

c) it is possible to put the inferences into systematic mathematical relation with other mathematical inferential practices

We claim that these are characteristic features of a diagrammatic practice in good order. The first two are related, in that the robustness of a diagrammatic practice to local deformation is the reason why it does not demand any special skill with pen, chalk or pointed stick. The third feature is most meaningful in cases like Euclidean geometry or knot theory where the diagrammatic practice came first. It offers a criterion for assessing a newly-invented diagram use, such as might be sketched on a napkin or

\footnotetext{
${ }^{36}$ Indeed, Feferman had no motive to provide such an analysis, because he thought that the underlying logical structure of every good proof is given by a derivation. His chief argument for this seems to be that otherwise, formal logic would lose its importance for philosophy of mathematics (2012:384-385).
} 
blackboard. It comes for free in cases where the diagram is a useful representation of already developed mathematics, and is usually trivial in published proofs. This is the case with the examples from higher dimensional algebra that David Corfield presents in section 10.4 of his (2003). In these cases, there is no need for a Manders-style analysis to show why the diagrammatic practice is rigorous, because it was designed to capture the rules of an already developed body of mathematics. Similarly, the inferential use of commutative diagrams, explored in De Toffoli (2017), obviously satisfies a) and c). In such cases, it is b) that offers insight into how they work.

In search of a Manders-style treatment of cases in contemporary mathematics, we considered papers by De Toffoli \& Giardino. These detailed and valuable studies make reference to spatial intuition and the examination and manipulation of mental models in their analyses of some proofs. This presents a difficulty, if we wish to offer a satisfying logical account of how these proofs work. However, reflection on these cases and work by Leitgeb and Feferman suggests that we can read such references to intuition and mental models as invitations to reconstruct an implied diagrammatic practice. Our three conditions above can then serve as success criteria for such a reconstruction. If a proof that appeals to mental models can be reconstructed diagrammatically such that it satisfies these three points, then its rigour can be analysed and evaluated. It may still be invalid, but the question of its validity or invalidity will not turn on unsharable inner intuitions.

In the philosophy of mathematical practice, it is important to pursue the analysis at an appropriate level of generality. With too narrow a focus, one passes from philosophy into history or journalism; too broad, and one loses touch with the variety (what Wittgentein called the 'motley') of proof practices. Here we concern ourselves only with proofs that involve diagrams, explicitly or implicity. Nevertheless, some of the ideas in play have wider application. As we saw, criterion c) is not restricted to diagrammatic practices, but is true of modern mathematics generally and can serve as a test for any putative mathematical practice. I have argued elsewhere ${ }^{37}$ that diagrammatic practices are just one kind of topic-specific inferential action-there are plenty of others. However, focussing on diagrammatic practices has the merit of bringing criteria a) and b) into view.

${ }^{37}$ Larvor (2012a) 


\section{References}

Auslander, Joseph (2008) 'On the Roles of Proof in Mathematics' In Proof and Other Enigmas, B. Gold and R. Simons, 61-77. Mathematical Association of America.

Avigad, J. 2008. Computers in Mathematical Inquiry. In The Philosophy of Mathematical Practice, ed. P. Mancosu, 302-316. Oxford: Oxford University Press.

Avigad, J., Dean, E and Mumma, J. (2009). A Formal System For Euclid's Elements. The Review of Symbolic Logic, 2, pp 700-768. doi:10.1017/S1755020309990098.

Azzouni, J. 2004. The Derivation-Indicator View of Mathematical Practice. Philosophia Mathematica (III) 12(2): 81-105.

Azzouni, J. 2009. Why do informal proofs conform to formal norms? Foundations of Science 14: 9-26.

Azzouni, J. 2013. The Relationship of Derivations in Artificial Languages to Ordinary Rigorous Mathematical Proof. Philosophia Mathematica (III) 21: 247-254.

Buldt, Bernd, Löwe, Benedikt \& Müller, Thomas "Towards a new epistemology of mathematics" Erkenntnis 68 (3):309 - 329 (2008)

Carter, J. (2010). Diagrams and proofs in analysis. International Studies in the Philosophy of Science, 24(1).

Corfield, D. (2003). Towards a Philosophy of Real Mathematics. Cambridge University Press.

Detlefsen, M. 2008. Proof: Its Nature and Significance. In Proof and Other Enigmas, B. Gold and R. Simons, 3-32. Mathematical Association of America.

De Toffoli, S. (2017). 'Chasing' the diagram - the use of visualizations in algebraic reasoning. The Review of Symbolic Logic, 10(1), 158-186.

De Toffoli, S. \& Giardino, V. (2016), 'Envisioning Transformations. The Practice of Topology'. In: Mathematical Cultures, edited by B. Larvor, Birkhäuser Science, Springer.

De Toffoli, S. \& Giardino, V. (2014), 'An Inquiry into the Practice of Proving in Low-Dimensional Topology'. Boston Studies in the History and the Philosophy of Science, 308, $315-336$.

De Toffoli, S. \& Giardino, V. (2013), 'Forms and Roles of Knot Diagrams'. Erkenntnis, 79(4), 829 842.

Feferman, S. (2012) "And so on...: reasoning with infinite diagrams" Synthese 186: 371. doi:10.1007/s11229-011-9985-6

Ferreirós, José (2016) Mathematical Knowledge and the Interplay of Practices Princeton University Press. 
Giardino, Valeria (2017) "L'imagination manipulatoire en mathématique», Bulletin d'Analyse Phénoménologique [En ligne], Volume 13, Numéro 2: L'acte d'imagination: Approches phénoménologiques (Actes $n^{\circ} 10$ )

Goethe, N. \& Friend, M. 2010. Confronting the Ideals of Proof with the Ways of Proving of the Research Mathematician. Studia Logica 96: 273-288.

Grosholz, Emily (2007) Representation and Productive Ambiguity in Mathematics and the Sciences. Oxford University Press.

Hales, T. C. (2012). Dense Sphere Packings : a Blueprint for Formal Proofs. Cambridge University Press.

Hardy, G. H. (1929) Mathematical Proof Mind, New Series, Vol. 38, No. 149 pp. 1-25.

Hersh, Reuben (1997) Prove-Once More and Again Philosophia Mathematica (III) 5(2): 153165.

Krön, B. (2010). Cutting up graphs revisited - a short proof of Stallings' structure theorem. Groups - Complexity - Cryptology, 2(2), pp. 213-221.

Lakatos, I. 1976. Proofs and Refutations. Ed. J. Worrall and E. Zahar. Cambridge: Cambridge University Press.

Larvor, Brendan (2012a) "How to think about informal proofs" Synthese 187 (2):715-730

Larvor, Brendan (2012b) "What Philosophy of Mathematical Practice Can Teach Argumentation Theory about Diagrams and Pictures" The Argument of Mathematics Aberdein, A. \& Dove, I. (eds). Springer pp. 209-222.

Larvor, Brendan (2016) "Why the naïve Derivation Recipe model cannot explain how mathematicians' proofs secure mathematical knowledge" Philosophia Mathematica.

Leitgeb, Hannes (2009) "On Formal and Informal Provability" in New Waves in Philosophy of Mathematics edited by Otávio Bueno and Øystein Linnebo. Palgrave Macmillan pp. 263-299

Löwe, Benedikt \& Müller, Thomas (2010) "Skills and mathematical knowledge" in: Benedikt Löwe, Thomas Müller, PhiMSAMP, Philosophy of Mathematics: Sociological Aspects and Mathematical Practice, London [Texts in Philosophy 11], pp. 265-280

Macbeth, Danielle (2015) "Reasoning in Mathematics and Machines: The Place of Mathematical Logic in Mathematical Understanding", AISB Convention 2015 Proceedings (online).

Mac Lane, S. 1986. Mathematics: Form and Function. New York: Springer. 
Manders, Ken (2008a) Diagram-based geometric practice. Mancosu, P., The Philosophy of Mathematical Practice, Oxford University Press 2008, pages 65-79, 2008.

Manders, Ken (2008b) The Euclidean diagram. Mancosu, P., The Philosophy of Mathematical Practice, Oxford University Press 2008, pages 80-133, 1995.

Manin, Yu. I. (1981) A Digression on Proof The Two-Year College Mathematics Journal, Vol. 12, No. 2, pp. 104-107

Mumma, John (2010) Proofs, pictures, and Euclid Synthese 175:255-287

Pelc, A. 2009. Why do we believe theorems? Philosophia Mathematica (III) 17(1): 84-94.

Pollard, Stephen (2014) review of Reuben Hersh's Experiencing Mathematics: what do we do when we do mathematics? In Philosophia Mathematica (III) 22(2): 271-274.

Rav, Y. 1999. Why Do We Prove Theorems? Philosophia Mathematica (III) 7(1): 5-41.

Rav, Y. (2007) A Critique of the Formalist-Mechanist Version of the Justification of Arguments in Mathematicians' Proof Practices. Philosophia Mathematica (III) 15(3): 291-320.

Robinson, J.A. 1997. Informal Rigor and Mathematical Understanding. In Computational Logic and Proof Theory. Proceedings of the $5^{\text {th }}$ annual Kurt Gödel Colloquium. Heidelberg \& New York: Springer.

Robinson, J.A. (2000) 'Proof = Guarantee + Explanation' in Intellectics and Computational Logic: Papers in Honor of Wolfgang Bibel. Dordrecht: Kluwer.

Russell, Bertrand (1902) The Teaching of Euclid, The Mathematical Gazette 2 (33), 165-167.

Shin, S.-J. (2004). Heterogeneous reasoning and its logic. The Bulletin of Symbolic Logic, 10(1), 86-106.

Starikova, Irina 2010 "Why do Mathematicians Need Different Ways of Presenting Mathematical Objects? The Case of Cayley Graphs" Topoi Volume 29, Issue 1, pp 41-51

Tanswell, F. 2015. A Problem with the Dependence of Informal Proofs on Formal Proofs. Philosophia Mathematica (III) 23(3): 295-310.

Thurston, W. 2006. On Proof and Progress in Mathematics. In 18 Unconventional Essays on the Nature of Mathematics, ed. R. Hersh, 37-55. New York: Springer.

Van Bendegem Jean Paul (1988) Non-Formal Properties of Real Mathematical Proofs. Proceedings of the Biennial Meeting of the Philosophy of Science Association, Vol. 1: Contributed Papers (1988), pp. 249-254. University of Chicago Press. 
Van Bendegem Jean Paul (1990) Characteristics of real mathematical proofs. A. Diaz, J. Echeverria en A. Ibarra (eds.), Structures in mathematical theories, Servicio Editorial Universidad del Pais Vasco, San Sebastian, pp.333-337.

Weir, Alan (2016). Informal Proof, Formal Proof, Formalism. The Review of Symbolic Logic, 9, Pp 23-43. Doi:10.1017/S1755020315000234.

Wittgenstein (1978) Remarks on the Foundations of Mathematics. Ed. G.H. von Wright, R. Rhees \& G.E.M. Anscombe, trans. G.E.M. Anscombe. Third edition. Oxford: Blackwell. 